\title{
PARÂMETROS COLORIMÉTRICOS NO SISTEMA CIELab PARA MADEIRAS DE FLORESTAS NATURAIS
}

Gabriella da Silva França1, Zaíra Morais dos Santos Hurtado de Mendoza ${ }^{2}$,

Pedro Hurtado de Mendoza Borges ${ }^{3}$, Vanessa Corrêa da Mata ${ }^{4}$, Esther Saraiva Carvalho de Souza ${ }^{5}$

1Bacharel em Engenharia Florestal da Universidade Federal de Mato Grosso (UFMT), Cuiabá-MT, Brasil.

2Professora Doutora do curso de Engenharia Florestal da Universidade Federal de

Mato Grosso (UFMT), Cuiabá-MT, Brasil. E-mail: zairamorais09@gmail.com ${ }^{3}$ Professor Doutor do curso de Agronomia da Universidade Federal de Mato Grosso (UFMT), Cuiabá-MT, Brasil.

${ }^{4}$ Mestranda em Ciências Florestais e Ambientais da Universidade Federal de Mato Grosso (UFMT), Cuiabá - MT, Brasil.

${ }^{5}$ Bacharel em Engenharia Florestal da Universidade Federal de Mato Grosso (UFMT), Cuiabá-MT, Brasil.

\section{Recebido em: 04/10/2019 - Aprovado em: 30/11/2019 - Publicado em: 15/12/2019} DOI: 10.18677/EnciBio_2019B43

\begin{abstract}
RESUMO
A cor da madeira é uma característica de grande importância para a indicação de uso da madeira e até mesmo na identificação das espécies florestais. Sua classificação pode influenciar e facilitar a escolha de madeiras que sejam mais atrativas esteticamente para o mercado, sem que seja necessária a utilização de corantes, valorizando assim, o uso da madeira em sua cor natural. Existem disponíveis atualmente equipamentos de alta precisão, fácil manuseio e que fazem a leitura da cor da madeira de maneira eficiente. São eles os colorímetros, sensores e espectrofotômetros, que vem paulatinamente substituindo métodos antigos e subjetivos. O presente estudo teve como objetivo comparar dois métodos de determinação de parâmetros de cor do sistema CIELab para 15 espécies de madeiras de florestas naturais. O material utilizado estava em forma de tábuas, que foram subdivididas em amostras menores de $15 \mathrm{~cm}$ de comprimento, $8 \mathrm{~cm}$ de largura e $1,5 \mathrm{~cm}$ de espessura, que foram lixadas para a retirada das imperfeições. Ao todo foram feitas 45 leituras exclusivamente no cerne das amostras. Para as leituras dos valores de luminosidade $\left(L^{*}\right)$ e as coordenadas cromáticas $a^{*}$ e $b^{*}$ do sistema CIELab utilizou-se o Colorímetro Minolta CR400 e o Sensor TCS34275. Na avaliação da luminosidade $\left(L^{*}\right)$ e das coordenadas cromáticas $a^{*}$, o Colorímetro Minolta CR400 apresentou maior precisão na distinção das cores quando comparado ao Sensor TCS34275. Porém, na avaliação das coordenadas cromáticas de $b^{*}$, ambos os métodos apresentaram resultados satisfatórios e equivalentes.
\end{abstract}

PALAVRAS-CHAVES: Arduino, colorímetro, madeira. 


\title{
COLORIMETRIC PARAMETERS IN CIELAb SYSTEM FOR NATIVE FOREST WOODS
}

\begin{abstract}
The color of the wood is a feature of great importance for the indication of use of the species and even identification when associated with other factors, such as the presence of extractive and tree age. Its classification can influence and facilitate the choice of species that are more aesthetically attractive to the market, without the use of dyes, thus enhancing the use of wood in its natural color. Nowadays, a high precision, easy-to-use equipment efficiently shows the wood color. They are the colorimeters, sensors and spectrophotometers, replacing old and subjective methods. The aim of this research was to compare two methods of determining CIELab color parameters for fifteen native forest wood species. The material used was in the form of planks, which were subdivided into samples smaller than $15 \mathrm{~cm}$ long, $8 \mathrm{~cm}$ wide and $1.5 \mathrm{~cm}$ thick, which were sanded to remove imperfections. In all, 45 readings were taken exclusively at the heart of the samples. The luminosity values $\left(L^{*}\right)$ and the chromatic coordinates $a *$ and $b$ * of the CIELab system were analyzed with two different equipment: the Minolta CR400 Colorimeter and the TCS34275 Sensor. To evaluate the brightness $\left(\mathrm{L}^{*}\right)$ and the chromatic coordinates $\mathrm{a}^{*}$, the Minolta CR400 Colorimeter showed greater accuracy in color distinction when compared to the TCS34275 Sensor. However, in the evaluation of the chromatic coordinates of $b$ *, both methods presented satisfactory and equivalent results.
\end{abstract}

KEYWORDS: Arduino, colorimeter, wood.

\section{INTRODUÇÃO}

As propriedades organoléticas da madeira são aquelas que podem ser percebidas pelos sentidos humanos, como cheiro, gosto, sabor, odor, textura e cor. Dentre elas, a cor é um dos parâmetros que valoriza comercialmente a madeira, e que exerce grande aceitação ou não, quando se trata da sua utilização como objeto de decoração. Por esta razão, é cada vez mais comum que se utilizem corantes artificiais para tingir as madeiras e dar a elas uma cor específica desejada, para que sejam comercializadas como madeiras de alto valor. Segundo Freitas et al. (2016) a cor da madeira é um parâmetro fundamental, que potencializa a utilização da espécie principalmente para fins decorativos. Alguns fatores intrínsecos da espécie florestal podem influenciar na cor da madeira, tais como composição química, anatomia, idade da árvore e fatores genéticos (MARTINS et al., 2015).

Nos estudos para avaliar as cores das madeiras, utiliza-se métodos subjetivos ou científicos. Os métodos subjetivos mais empregados são a visualização direta da amostra e a carta de Munsell. Nessa carta, a cor da amostra é verificada em uma escala comparativa com o padrão de cores para solos. Cada padrão corresponde a uma notação Munsell que é feita com base no matiz, valor e croma. A matiz é o comprimento de onda da luz, o valor é o brilho ou a tonalidade, e o croma é a intensidade ou pureza da cor em relação ao cinza. Conforme Silva et al. (2015) a atribuição da coloração de uma madeira pela análise visual é bastante subjetiva, visto que a mesma pode ser diferente de acordo com o observador e a luminosidade

Visando contornar a subjetividade na análise de cores, foram desenvolvidos métodos científicos quantitativos que empregam variáveis numéricas para as interações provocadas pela luz em uma superfície. Nas análises científicas, o 
colorímetro é um dos equipamentos mais utilizados, e tem como princípio aumentar a percepção das cores pelos olhos humanos, através do emprego de um sistema denominado CIELab (MENDOZA; BORGES, 2015).

Nesse sistema, o valor de luminosidade $L^{*}$ é aproximadamente $o$ valor da luminância $Y$ (para o $\mathrm{CIE} x y \mathrm{Y}$ ) variando de branco a preto, assumindo o valor 0 (zero) para o preto absoluto e 100 para o branco total. O valor de $a^{*}$ pode variar de verde a vermelho e 0 valor de $b^{*}$ pode variar de azul a amarelo, analogamente à percepção das cores pelo cérebro, ou seja, cores-opostas. O sistema de medição de cores CIELab 1976 é baseado em três elementos: a luminosidade ou claridade, a tonalidade ou matiz e a saturação ou cromaticidade (BARROS, 2014).

A colorimetria quantitativa é uma metodologia bastante objetiva e muito eficaz quando se diz respeito à medição da cor da madeira. Existem dois tipos de aparelhos apropriados para descrever numericamente cada elemento da composição de uma cor em superfícies, os colorímetros e os espectrofotômetros. Além disso, a utilização de técnicas não destrutivas como a colorimetria e a espectrofotometria vem ganhando espaço, devido à possibilidade de analisar um grande volume de material com maior rapidez e uma alta precisão, tornando o procedimento versátil, enquadrando-se com facilidade em uma rotina de linha de produção e por ser uma técnica não destrutiva, ela permite a reutilização da peça testada (PAULA et al., 2016). Apesar de todas essas vantagens os calorímetros e os espectrofotômetros, apresentam custos elevados de aquisição e manutenção, o que inviabiliza em parte, o seu uso. Porém, devido à crescente necessidade em automatizar os processos de produção, para aumentar a produtividade e economizar gastos, existem no mercado plataformas de prototipagem eletrônica de baixo custo, que oferecem amplas possibilidades para desenvolver tecnologias acessíveis, possibilitando o uso de sensores disponíveis no mercado e que possuem uma linguagem de programação simples. Esse é o caso, por exemplo, dos sensores de cores RGB, que já são amplamente utilizados no setor agrícola, alimentício e comércio. $O$ princípio de funcionalidade deles baseia-se em três cores primárias (Red, Green e Blue - vermelho, verde e azul) a partir das quais consegue-se identificar diversas outras cores.

Indo ao encontro dessas necessidades, o presente estudo teve como objetivo comparar dois métodos científicos de determinação de cores em madeiras serradas, provenientes de florestas naturais, tendo como referência o sistema CIELab.

\section{MATERIAL E MÉTODOS}

A coleta do material foi realizada no depósito unificado de madeira da SEMA/MT, na cidade de Cuiabá - MT, CEP 78098-500, coordenadas geográficas $15^{\circ} 39^{\prime} 40.1^{\prime \prime S} 55^{\circ} 58^{\prime} 26.9 " \mathrm{~W}$. Aleatoriamente foram escolhidas quinze espécies florestais, que estavam dispostos em pilhas de madeira serrada, e que já haviam sido previamente identificados pelos peritos, ao nível de gênero e/ou espécie (Tabela 1). 
TABELA 1. Identificação botânica das espécies utilizadas no estudo.

\begin{tabular}{llll}
\hline Nome Vulgar & Gênero & Ordem & Família \\
\hline Angelim Amargoso & Vatairea sp. & Fabales & Fabaceae \\
Castanha Jarana & Lecythis sp. & Ericales & Lecythidaceae \\
Muiracatiara & Astronium sp. & Sapindales & Anacardiaceae \\
Cambará & Qualea sp. & Myrtales & Vochysiceae \\
Cupiúba & Goupia glabra & Malpighiales & Goupiaceae \\
Tauari & Couratari sp. & Ericales & Lecythidaceae \\
Angelim Pedra & Hymenolobium sp. & Fabales & Fabaceae \\
Cedrinho & Erisma uncinatum & Myrtales & Vochysiceae \\
Pau Roxinho & Peltogyne sp. & Fabales & Fabaceae \\
Jequitibá & Cariniana sp. & Ericales & Lecythidaceae \\
Itaúba & Mezilaurus itauba & Laurales & Lauraceae \\
Ipê & Tabebuia sp. & Lamiales & Bignoniaceae \\
Faveira Dura & Enterolobium sp. & Fabales & Fabaceae \\
Garapeira & Apuleia sp. & Fabales & Fabaceae \\
Ucuubarana & Iryanthera sp. & Magnoliales & Myristicaceae \\
\hline
\end{tabular}

Fonte: INDEA/MT (2019)

\section{Preparo dos corpos de prova}

Após a identificação, escolheu-se no interior da pilha uma tábua de cada espécie e em seguida efetuou-se a retirada de amostras menores com dimensões de $15 \mathrm{~cm}$ de comprimento, $8 \mathrm{~cm}$ de largura e $1,5 \mathrm{~cm}$ de espessura, sendo todas as amostras lixadas para retirada das impurezas.

Uma parte dessas amostras foi utilizada para as análises de cores e a outra parte foi empregada para a xiloteca, ampliando-se assim, o acervo de madeiras da Faculdade de Engenharia Florestal.

Os ensaios de cores foram realizados empregando-se o colorímetro Minolta CR400 e o Sensor TCS34275. As cores nas madeiras foram verificadas exclusivamente no cerne das amostras, em uma das faces tangenciais (escolhendose a que apresentava a maior porcentagem de cerne) e em 3 locais distintos, subdivididos de cinco em cinco centímetros, e demarcados ao longo da amostra como lateral direita, meio e lateral esquerda. Ao todo foram realizadas 45 leituras com o colorímetro e 45 leituras com o Sensor $(15 \times 3=45)$.

As análises foram feitas primeiramente no colorímetro, o qual gerou as informações sobre as cores diretamente no sistema CIELab. Antes de iniciar as leituras, realizou-se a calibragem utilizando-se uma placa de cerâmica branca, denominada $\mathrm{cr}-\mathrm{a} 43$, que acompanha 0 equipamento, cuja escala padrão são: $z=$ 93,$6 ; x=0,3133 ; y=0,3195$. O ensaio foi executado de forma ininterrupta, em um ambiente com luz artificial uniforme, sem a incidência de luz natural.

$\mathrm{Na}$ sequência foram efetuados os ensaios com o sensor TCS34275, dispositivo eletrônico fabricado pela Adafruit, capaz de detectar os níveis das cores vermelho, verde e azul presentes em objetos, e os converter num valor numérico na escala RGB (vermelho, verde e azul). Entretanto, esses valores não coincidem exatamente com o intervalo entre 0 e 255 para os códigos RGB disponíveis nos computadores e seus programas. Diante disso, optou-se por calibrar o sensor 
utilizando-se paleta de cores disponíveis em meios digitais, e posteriormente realizou-se o ajuste de modelos empregando-se análise de regressão.

Inicialmente, o sensor foi acoplado a um microcontrolador do tipo Arduino UNO. Para aprimorar a coleta dos dados, adicionou-se à placa do microcontrolador o módulo RTC3231, com a finalidade de controlar a data e o horário de coleta, bem como o módulo SD para cartão de memória, responsável pelo armazenamento dos dados. Logo após, realizou-se a calibragem com o propósito de compatibilizar os valores lidos pelo sensor com a escala RGB dos programas computacionais, o que complementaria a sua aplicação na área de colorimetria.

Para a calibração foram distribuídas aleatoriamente amostras de diferentes cores de papel cartão a 30 observadores voluntários. Os observadores identificaram os códigos RGB das amostras com auxílio da paleta de cores do programa PAINT, acessório do WINDOWS.

Em seguida, procedeu-se à leitura das amostras de papel cartão pelo sensor, formando-se 30 pares distintos de pontos ordenados. Com os dados obtidos realizou-se a análise de regressão com a finalidade de definir as equações de calibração 1, 2 e 3 para cada código R, G e B, respectivamente.

$$
\begin{aligned}
& V_{\text {Sensor }}=60,41 \cdot \mathrm{e}^{0,0063 \cdot V_{\text {Observador }}} \\
& V_{\text {Sensor }}=63,46 \cdot \mathrm{e}^{0,0078 \cdot V_{\text {Observador }}} \\
& V_{\text {Sensor }}=79,37 \cdot \mathrm{e}^{0,0062 \cdot V_{\text {Observador }}}
\end{aligned}
$$

\footnotetext{
Em que,

$\mathrm{V}_{\text {Sensor }}=$ Valor do código lido pelo sensor (adimensional);

$\mathrm{V}_{\text {Observador }}=$ Valor do código lido pelo observador (adimensional).
}

As curvas das equações citadas acima bem como os seus coeficientes de determinação $\left(R^{2}\right)$ apresentam-se na Figura 1. De acordo com essa figura observase que houve um adequado ajuste entre os valores lidos pelo sensor e pela percepção visual dos observadores, o que pode ser comprovado pelos valores de coeficiente de determinação superiores a 0,70. 

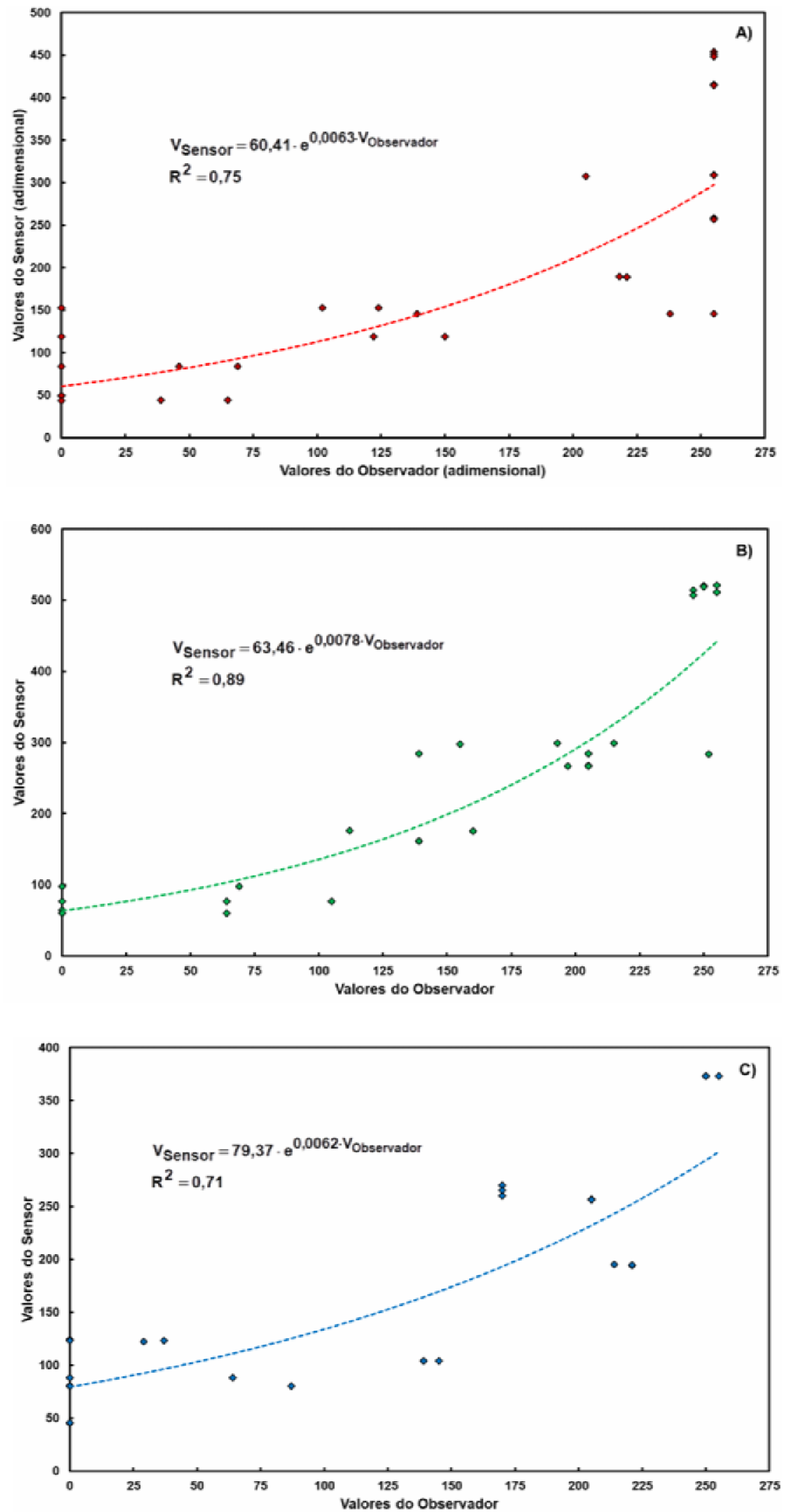

FIGURA 1. Curvas e equações de calibração do sensor TCS34275.

a) Escala R (vermelho); b) Escala G (verde); c) Escala B (azul). 
Os ensaios com o sensor foram efetuados nas mesmas amostras de madeira e com a mesma metodologia adotada para o colorímetro. Entretanto, para fins de uniformidade, os dados obtidos na escala RGB (Sensor) foram convertidos para CIELab (escala do colorímetro). Esse procedimento possibilitou a comparação dos resultados entre ambos os equipamentos, aplicando-se o Teste t (Student) em nível de $5 \%$ de probabilidade.

\section{RESULTADOS E DISCUSSÃO}

Os valores médios das variáveis encontradas para o sistema CIE L*a*b* e o teste t (Student) para as médias analisadas, encontram-se na Tabela 2.

TABELA 2. Teste $t$ (Student) para as variáveis do sistema CIElab obtidas conforme os equipamentos utilizados.

\begin{tabular}{|c|c|c|c|c|c|c|}
\hline \multirow{3}{*}{$\begin{array}{l}\text { Parâmetros } \\
\text { do teste t } \\
\text { (Student) }\end{array}$} & \multicolumn{6}{|c|}{ Valores médios das variáveis no sistema CIELab } \\
\hline & \multicolumn{2}{|c|}{$L^{*}$} & \multicolumn{2}{|c|}{$a^{*}$} & \multicolumn{2}{|c|}{$b^{*}$} \\
\hline & Color. & Sensor & Color. & Sensor & Color. & Sensor \\
\hline Média & 55,60 & 29,60 & 8,04 & $-0,42$ & 15,83 & 15,12 \\
\hline Variância & 71,82 & 135,31 & 8,31 & 8,31 & 29,93 & 30,30 \\
\hline Graus de Liberdade & \multicolumn{2}{|c|}{88} & \multicolumn{2}{|c|}{88} & \multicolumn{2}{|c|}{88} \\
\hline Estatística do teste & \multicolumn{2}{|c|}{12,11} & \multicolumn{2}{|c|}{13,92} & \multicolumn{2}{|c|}{0,61} \\
\hline Probabilidade & \multicolumn{2}{|c|}{$9,3010^{-21}$} & \multicolumn{2}{|c|}{$2,8310^{-24}$} & \multicolumn{2}{|c|}{0,30} \\
\hline Valor crítico & \multicolumn{2}{|c|}{1,67} & \multicolumn{2}{|c|}{1,67} & \multicolumn{2}{|c|}{1,67} \\
\hline Observações & \multicolumn{2}{|c|}{45} & \multicolumn{2}{|c|}{45} & \multicolumn{2}{|c|}{45} \\
\hline
\end{tabular}

Para a variável luminosidade $\left(\mathrm{L}^{*}\right)$, o valor crítico foi menor do que a estatística do teste com probabilidade muito inferior a 0,05 $\left(9,3010^{-21}\right)$, rejeitando-se assim a hipótese inicial de que ambas as variáveis são equivalentes para os dois equipamentos. $\mathrm{Na}$ análise das coordenadas cromáticas da posição do ponto de cor sobre o eixo verde-vermelho $\left(\mathrm{a}^{*}\right)$, o valor da probabilidade foi muito inferior a 0,05 $\left(2,8310^{-24}\right)$, rejeitando-se também a hipótese inicial de equivalência para os dois equipamentos, ou seja, são distintos.

$\mathrm{Na}$ coordenada cromática correspondente ao eixo azul-amarelo $\left(b^{*}\right)$, situado em posição perpendicular com o eixo verde-vermelho $\left(a^{*}\right)$ no plano da cor, o valor crítico foi superior à estatística do teste com probabilidade maior do que $0,05(0,30)$, aceitando-se assim a hipótese inicial, ou seja, os valores obtidos por ambos os equipamentos são equivalentes e atingiram valores muito próximos, sendo considerados semelhantes estatisticamente.

Com base nos resultados, constata-se que, apenas para os parâmetros de luminosidade $\left(L^{*}\right)$ e a coordenada cromática de $a^{*}$, houve diferenças significativas, 0 que indica a não equivalência das cores lidas por ambos os equipamentos. Como a variável $L^{*}$ indica uma variação entre tons mais claros e mais escuros, e a variável a* a variação entre os tons vermelhos e verde, deduz-se que para estas cores em específico, houve diferença significativa entres os valores obtidos, o que não ocorreu com os valores de $b^{*}$, que variaram entre os tons de amarelo e azul, sendo nesse caso, equivalente para ambos os equipamentos utilizados. Nesse caso tendendo para o amarelo $(+b)$, comprovando assim a tonalidade predominante das madeiras, razão pela qual essa variável apresentou a maior precisão. 
As discrepâncias entre as leituras dos dois equipamentos podem ter ocorrido, possivelmente, devido à presença de diferentes tons na madeira, que nesse caso, se mostraram não sensíveis e/ou pouco sensíveis ao Sensor TCS34275. Também, a luz artificial pode ter interferido significativamente, pois o sensor possui um filtro infravermelho para compensar a influência da radiação proveniente da iluminação e neste caso a captação das cores pelo colorímetro pode ter sido prejudicada. Devese ressaltar, ainda, que a calibração do sensor teve a influência da subjetividade dos observadores para identificar com precisão as cores das amostras de papel, pois há uma região muito confusa no eixo verde-vermelho $\left(a^{*}\right)$, principalmente, no intervalo entre -10 (verde) e +10 (vermelho), na qual as cores não apresentaram clareza.

As análises realizadas prioritariamente no cerne sugerem que as variações detectadas nas variáveis $L^{*}$ e $a^{*}$ estejam ligadas à presença de extrativos na madeira. Garcia et al. (2016), em um estudo sobre a variação da cor da madeira de teca em função da densidade e do teor de extrativos, observaram que árvores de plantios menos densos (com espaçamentos maiores que $6 \times 2 \mathrm{~m}$ ), produziram madeiras com maiores teores de extrativos no cerne quando comparado ao alburno. Os mesmos autores verificaram também que nas condições citadas acima, o teor de extrativos apresentou uma correlação significativa com a luminosidade $\left(L^{*}\right)$ e a tonalidade vermelha $\left(a^{*}\right)$, indicando que as madeiras mais escuras (menor $L^{*}$ ) e com mais pigmento vermelho evidenciaram um maior teor de extrativos. Romagnoli et al. (2013), trabalhando com determinação de cores em madeira de Tabebuia serratifolia, verificaram que os níveis do parâmetro b* também estiveram associados aos teores de determinados compostos extraíveis da madeira, mais especificamente a algumas naftoquinonas, como o lapachol e a desidro $\alpha$-lapachona. Sendo assim, em razão da maior impregnação de diversas substâncias responsáveis pela coloração da madeira principalmente no cerne, sugere-se que as variações obtidas para as variáveis $L^{*}, a^{*}$ e $b^{*}$, estejam ligadas diretamente à presença de extrativos no cerne das madeiras.

Nesse contexto, ressalta-se a importância de utilizar equipamentos que sejam precisos para avaliar a cor das madeiras, seja através de sensores ou colorímetros, visando a valorização das madeiras nativas. Além disso, o conhecimento sobre a cor da madeira poderá fornecer informações capazes de subsidiar uma pré-classificação das madeiras para a combinação de cores em tons variados, considerando-se a preferência de mercado, o que agregaria valor a elas. A determinação dos parâmetros das cores de madeiras poderá também fomentar um banco de dados de cores, para a padronização de uma paleta de cores de madeiras nativas, facilitando assim a comercialização e utilização dessas madeiras.

\section{CONCLUSÃO}

Para avaliar a luminosidade $\left(\mathrm{L}^{*}\right)$, que determina os tons mais claros e mais escuros e as coordenadas cromáticas $a^{*}$, que representam a variação de tons do verde ao vermelho, o Colorímetro Minolta CR400 apresentou maior precisão na distinção das cores quando comparado ao Sensor TCS34275. Porém, na avaliação das coordenadas cromáticas de $b^{*}$, que correspondem aos tons que variam entre amarelo e azul na escala CIELab, ambos os equipamentos se mostraram eficazes para sua determinação. Contudo, o sensor apresentou-se como alternativa viável e econômica, embora seja necessário realizar, ainda, mais estudos na área como por exemplo, ajustes e calibrações. Isso poderia ser melhorado utilizando-se cores mais 
definidas ampliando-se o universo de matizes e tons para auxiliar o observador, visando elevar a precisão do equipamento.

\section{REFERÊNCIAS}

BARROS, S. V. S.; MUNIZ, G.I.B.; MATOS, J. L. M. caracterização colorimétrica das madeiras de três espécies florestais da Amazônia. Cerne, v.20, n. 3, p. 337-32, 2014.

Disponível em: http://www.scielo.br/pdf/cerne/v20n3/a01v20n3.pdf DOI: 10.1590/01047760201420031421

FREITAS, A. S.; GONCALEZ, J. C.; DEL MENEZZI, C. H. Tratamento Termomecânico e seus Efeitos nas Propriedades da Simarouba amara (Aubl.). Floresta Ambiente. vol.23, n.4, p. 1-8. 2016.

Disponível em: <http://www.scielo.br/scielo.php?script=sci_arttext\&pid=S2179$80872016000400565 \&$ lng $=$ pt\&nrm=iso $>$.

DOI: $10.1590 / 2179-8087.144115$

GARCIA, R. A.; MARINONIO, G. B., Variação da Cor da Madeira de Teca em Função da Densidade e do Teor de Extrativos. Floresta Ambiente, Seropédica, v. 23, n. 1, p. 124-134, mar. 2016.

Disponível em: https://floram.org/article/10.1590/2179-8087.035313/pdf/floram-23-1124.pdf.

DOI: http://dx.doi.org/10.1590/2179-8087.035313.

LIMA, C. M.; GONÇALEZ, J. C.; COSTA, T. R. V.; PEREIRA, R. S.; LIMA, J. B. M.; LIMA, M. S. A. Comportamento da cor de lâminas de madeira de pau-marfim (Balfourodendron riedelianum) tratada com produtos de acabamento. Revista Árvore, v.37, n.2, p.377-384, 2013.

Disponível em: http://www.scielo.br/pdf/rarv/v37n2/20.pdf

DOI: http://dx.doi.org/10.1590/S0100-67622013000200020

MARTINS, M. F.; BELTRAME, R.; DELUCIS, R. A.; GATTO, D. A; CADEMARTORI, P. H. G.; SANTOS, G. A. Colorimetria como ferramenta de agrupamento de madeira de clones de eucalipto. Pesquisa Florestal Brasileira. v.35, n. 84, p. 1-8, 2015. Disponível em: https://pfb.cnpf.embrapa.br/pfb/index.php/pfb/article/view/929 DOI: 10.4336/2015.pfb.35.84.929 e-ISSN: 1983-2605.

MENDOZA, Z. M. S. H.; BORGES, P. H. M. Análise colorimétrica do extrato aquoso de folhas de teca. Revista Árvore, v.39, n.5, p.953-961. 2015.

Disponível em: http://www.scielo.br/pdf/rarv/v39n5/0100-6762-rarv-39-05-0953.pdf DOI: http://dx.doi.org/10.1590/0100-67622015000500018.

PAULA, M. H.; MESQUITA, R. R. S.; GONÇALEZ, J. C.; RIBEIRO, E.S.; SOUZA, S. R. Utilização de métodos não destrutivos para caracterização simplificada da madeira de cumaru (Dipteryx odorata Willd). Biodiversidade, v.15, n.2, p. 136-149, 2016.

Disponível em:

http://periodicoscientificos.ufmt.br/ojs/index.php/biodiversidade/article/view/3967 
ROMAGNOLI, M.; SEGOLONI, E.; LUNA, M.; MARGARITELLI, A.; GATTI, M.; SANTAMARIA, U.; VINCIGUERRA, V. Wood colour in Lapachol (Tabebuia serratifolia): chemical compositionand industrial implications. Wood Science and Technology, New York, v. 47, n. 4, p. 701-716, 2013.

Disponível em: https://link.springer.com/article/10.1007/s00226-013-0534-y DOI: 10.1007/s00226-013-0534-y

SILVA, E. S.; STANGERLIN, D. M.; GATTO, D. A.; CALEGARI, L; PARIZ, E. Colorimetria da madeira de oito espécies nativas do estado do Rio Grande do Sul, Brasil. Ciência da Madeira (Brazilian Journal of Wood Science), v. 6, n. 1, p. 31-37, 2015.

Disponível em:

https://periodicos.ufpel.edu.br/ojs2/index.php/cienciadamadeira/article/view/4292/411

DOI: 10.12953/2177-6830/rcm.v6n1p31-37 